\title{
ASSESSMENT OF UNSTEADINESS MODELLING FOR TRANSIENT NATURAL CONVECTION
}

\section{Fadl (1)}

Department of Engineering Science University of Oxford, Oxford, UK,

L. He ${ }^{(2)}$

Department of Engineering Science University of Oxford, Oxford, UK

\section{P. Stein}

GE Power, 5400 Baden, Switzerland

\section{G. Marinescu}

GE Power, 5400 Baden, Switzerland

(1, currently: CREST, Loughborough University, email: $\underline{\text { m.s.fadl@lboro.ac.uk) }}$

(2, corresponding author, email: Li.He@eng.ox.ac.uk)

\section{ABSTRACT}

Turbine flexible operations with faster startups/shutdowns are required to accommodate emerging renewable power generations. A major challenge in transient thermal design and analysis is the time scale disparity. For natural cooling, the physical process is typically in hours, but on the other hand, the time step sizes typically usable tend to be very small (sub-seconds) due to the numerical stability requirement for natural convection as often observed. An issue of interest is what time step sizes can and should be used in terms of stability as well as accuracy?

In this work, the impact of flow temporal gradient and its modelling is examined in relation to numerical stability and modelling accuracy for transient natural convection. A source term based dualtiming formulation is adopted, which is shown to be numerically stable for very large time steps. Furthermore, a loosely coupled procedure is developed to combine this enhanced flow solver with a solid conduction solver for solving unsteady conjugate heat transfer problems for transient natural convection. This allows very large computational time steps to be used without any stability issues, and thus enables to assess the impact of using different time step sizes entirely in terms of a temporal accuracy requirement. Computational case studies demonstrate that the present method can be run stably with a markedly shortened computational time compared to the baseline solver. The method is also shown to be more accurate than the commonly adopted quasi-steady flow model when unsteady effects are non-negligible. 


\section{1- INTRODUCTION}

The growing shares of renewable energy sources in the market and emerging solar power applications have set higher requirements on steam turbine operations. In order to design reliably a steam turbine for flexible operations, it is essential to understand dynamic thermal characteristics of steam turbine components during a transient process. Nowadays a power plant's competitiveness is measured not only on the performance per se but also on the overall plant operational economy. This includes the ability to start up and shut down quickly, and frequently for some applications, while having an adequate high cycle thermal fatigue life. For instance, a cooling down process will have to take into account material strength limits and usage factors [1], [2], and the residual temperature gradients when restarted may have some considerable impact on turbine life span. An adequate prediction of component metal temperatures before a restart becomes increasingly important, and is one of the key capabilities required for a transient steam turbine cooling process. Note also that steam turbines in solar power plants would face many more startups and shutdowns compared to typical base-load machines.

A number of efforts have been made to develop modelling based methods for prediction of turbine shutdown and cooling processes. Marinescu et al $[3,4]$ developed a new procedure for assessing the natural cooling of steam turbine components, validated with experimental measurements. The numerical cooling calculation is effectively based on a concept to approximate the fluid gross buoyancy during a natural cooling by an equivalent fluid conductivity, calibrated against experimental data. The procedure, though with notable empiricism, was shown to work quite well for natural cooling predictions of steam turbines.

In terms of higher fidelity CFD based convective heat transfer analyses for turbine applications, most commonly used procedures have been based on heat transfer coefficient (HTC), with an assumption that HTC is largely invariant with respect to wall temperature. A direct HTC based approach could be in considerable errors under certain conditions for forced convection heat transfer in a gas turbine blade passage, e.g. as observed by Maffulli and He, [5] [6], Zhang and He [7]. For a natural convection problem of the present interest, the near-wall flow is dominantly driven by the wall temperature, making a HTC based approach even more difficult. This strong dependence of flow on the wall thermal condition in natural convection presents a clear case for pursuing conjugate heat transfer (CHT) solutions for coupled fluid-solid domains.

For general CHT method developments and applications, the time scale disparity between fluid and solid parts as a fundamental feature must be recognized. The ratio between the two-time scales can be up 
to $10^{4}$ for gas turbine blades, as pointed out by He and Oldfield [8]. This time scale disparity presents a challenge for numerical solution methods adopted for the two domains, as well as for the interface treatment between them. A direct simultaneous coupling is a seemingly straightforward option, but can be hugely time consuming. On the other hand, the time scale disparity can also serve as a justification for using a quasi-steady flow model for transient CHT, i.e. coupling an unsteady solid solver with a steady fluid solver at each physical time step. The quasi-steady flow based loosely coupled CHT methods have been actively developed and applied to forced convection heat transfer problems for turbine configurations and others, e.g. [9], [10], [11]. For natural convection, there have been some attempts to use the direct coupled CHT solutions, e.g. [2] [12], [13], known to be computationally time consuming. However, the present authors are not aware of any published efforts of using a loosely coupled CHT approach for natural convection.

A particularly relevant issue as has been observed is related to the time step size allowable in natural convection simulations with common commercial codes (e.g. FLUENT). The numerical stability requirement tends to restrict the time step size to a very small value (typically sub-seconds), e.g. [12], [13], [14]. When a transient CHT solution for turbine flexible operations is pursued, this time step limit can be particularly restrictive, given the time scale disparity between the fluid and solid domain.

The present effort is chiefly motivated to investigate the applicability of a URANS based loosely coupled CHT method for transient natural convection. Central to this study is the impact of unsteadiness and its modelling in the context of transient natural convection at conditions relevant to steam turbine flexible operations. There are several related questions in this regard:

i) First of all, how restrictive is the time step limit in common commercial solvers?

ii) If, as will be shown later, a modified flow solver can work stably for much larger time steps, how large should a time step be for adequate time accuracy then?

iii) In relation to the common quasi-steady assumption adopted in previous loosely coupled CHT methods for forced convection, what is its impact on both accuracy and computational efficiency for natural convection?

The presentation of the computational studies is organized to help identify and understand the issues by contrasting two scenarios: a fluid-domain only problem versus a fluid-solid coupled CHT problem. After the baseline and the present solvers are introduced, two kinds of analyses are presented. We first consider the natural convection in a fluid domain only with a prescribed isothermal wall condition. The 
computational case studies for the fluid-domain will highlight the problem of the time step restriction of the baseline flow solver. After the case studies to underline the problem statement, the baseline solution results are then contrasted with the present source term based dual time stepping solver enabling a much larger time step. The results of the present solver with different time step sizes are then compared to identify the limits posed by the temporal accuracy. Furthermore, an assessment on the quasi-steady model is also included. Following the fluid-domain only results, transient CHT analyses are then presented. Again, the usable time step sizes in relation to the stability and accuracy requirement are examined, and the implications of the present results will also be discussed.

\section{METHODS FOR ANALYSES}

All the analyses are carried out based on a common commercial code FLUENT. The baseline methods are simply those of FLUENT as they are. The present methods are implemented by adding source terms to standalone flow and solid solvers from FLUENT and linking them via the User-Defined Function (UDF), as will be described. The overall unsteady conjugate heat transfer method has been recently developed based on a multi-scale framework methodology proposed by He and Fadl [15], validated and demonstrated for a range of transient forced convection cases. It is however the first time that such a loosely coupled CHT procedure is adopted for natural convection problems.

\section{2-1 Baseline Flow Solver for Direct Coupled CHT}

The flow is governed by the $3 \mathrm{D}$ unsteady compressible flow equations, in a short form.

$$
\frac{\partial U}{\partial t}+R_{f}(U)=S_{b}(U)
$$

where $U$ is the vector of conservative variables and $R_{f}$ is the standard residual for forced convection computations. It is noted that the source term for the buoyancy effect $S_{b}$ is added and listed separately, as it is the treatment of this term which does have a very significant impact on the usable time step due to numerical stability. In all the present studies, regardless of numerical procedures, we adopt the full

buoyancy term due to gravitational acceleration. Thus, the term is directly proportional to fluid density in line with the fully compressible flow model adopted.

For turbulent flows at high Rayleigh number conditions, the flow governing equations become the Reynolds-averaged Navier-Stokes equations (RANS for steady flows or URANS for unsteady flows), 
more precisely the density weighted (Favre) time averaged equations. In the present studies, the k- $\varepsilon$ two equation model is used for turbulence closure.

For unsteady flows, a pseudo time $\tau$ can be introduced for local sub-iterations by time-marching at each physical step. A dual timing form with an implicit source term treatment should be in a form (for a physical time step $\mathrm{N}$ ):

$$
\left(\frac{\partial \mathbf{U}}{\partial \tau}\right)^{N, k}+\frac{1.5 \mathbf{U}^{N, k}-2 \mathbf{U}^{N-1}+0.5 U^{N-2}}{\Delta t}+R_{f}\left(\mathbf{U}^{N, k-1}\right)=S_{b}\left(U^{N, k}\right)
$$

where $\mathrm{k}$ is the index of the sub-iterations. When the sub-iterations converge, the pseudo time term vanishes, and the full 2nd order temporal approximation of the unsteady flow equation (Eq.1) fully recovers.

For a solid domain, the temperature field is governed by the unsteady conduction equation.

$$
\frac{\partial T}{\partial t}+R_{S}(T)=0
$$

which is effectively in the same form as the unsteady fluid energy equation with zero velocity, but also with all fluid properties being replaced by those of the solid. The semi-discrete unsteady conduction equation in the dual timing form for time step $\mathrm{N}$ and sub-iteration $\mathrm{k}$ is:

$$
\left(\frac{\partial T}{\partial \tau}\right)^{N, k}+\frac{1.5 T^{N, k}-2 T^{N-1}+0.5 T^{N-2}}{\Delta t}+R_{S}\left(T^{N, k-1}\right)=0
$$

When a directly coupled CHT problem is solved, the physical conditions of temperature and heat flux continuity [16] between the fluid side ('f') and solid side ('S') are:

$$
\begin{aligned}
& q_{S}\left(T_{S}\right)^{N, k}=q_{f}\left(T_{f}\right)^{N, k} \\
& T_{S}^{N, k}=T_{f}^{N, k}
\end{aligned}
$$

A discretized form of Eq (5a), making use of Eq (5b), leads to an explicit expression of the wall temperature, so that the wall temperature is updated at each iteration, enabling the solutions on both sides to be marched forward concurrently.

\section{2-2 Present Source Term Based Unsteady Flow Solver for Loosely Coupled CHT}

The present methodology has been implemented in the commercial code FLUENT facilitating the UDF. The overall approach is to convert an unsteady CHT problem to a steady-like problem. The basic intent stems chiefly from the observation that unsteady natural convection solutions are subject to severe time step restriction for convergence due to numerical instabilities (as shown later), whilst neither does a 
steady natural convection nor an unsteady forced convection solution seem to have problems with convergence.

Consider the fluid domain first. We take a standard stand-alone fluid solution method for a steady natural convection problem as the baseline solver. Unsteady solutions are enabled completely through an extra source term explicitly added through UDF. The corresponding dual timing form of the fluid equation for physical time step $\mathrm{N}$ and sub-iteration step k becomes:

$$
\left(\frac{\partial \mathbf{U}}{\partial \tau}\right)^{N, k}+R_{f}\left(\mathbf{U}^{N, k-1}\right)=S_{b}\left(U^{N, k-1}\right)-\left(\frac{1.5 \mathbf{U}^{N, k-1}-2 \mathbf{U}^{N-1}+0.5 U^{N-2}}{\Delta t}\right)_{U D F}
$$

Note that both the physical temporal gradient term and the gravitational buoyancy term are in an explicit form, directly contributing to the balance of the unsteady flow equations when the pseudo-time term vanishes. A general dual timing formulation is known to be stable for large time steps due to the implicit backward difference for the physical temporal term, after Jameson [17]. It has also been widely used for URANS applications for unsteady turbomachinery flows with much larger physical time steps than that dictated by the Courant-Friedrich-Levy (CFL) number restricted numerical stability (e.g. Arnone et al [18], He [19]). It is noted that the dual timing procedure may require the physical time step to be smaller than that limited by CFL. This small-time step restriction, though not of concern for the present work, can be effectively overcome by simply limiting the pseudo time step [18]. As will be illustrated by the present results, this seemingly simple treatment of the physical temporal gradient completely as a source term, which is explicitly updated after each sub-iteration by UDF, is shown to have a clear and significant impact on the usable time step size for natural convection.

Similarly, for a solid domain, a stand-alone steady conduction solver is adopted. An unsteady conduction solution is also enabled by a source term by UDF, which is updated after each sub-iteration. The corresponding dual timing form for time step $\mathrm{N}$ and sub-iteration $\mathrm{k}$ is:

$$
\left(\frac{\partial T}{\partial \tau}\right)^{N, k}+R_{S}\left(T^{N, k-1}\right)=-\left(\frac{1.5 T^{N, k-1}-2 T^{N-1}+0.5 T^{N-2}}{\Delta t}\right)_{U D F}
$$

In a loosely coupled CHT system, the fluid solution and the solid solution exchange information across the interface only after a number sub-iterations are completed. Each exchange between the two domains is called a CHT coupling cycle. A simple and stable interface treatment is to specify a wall temperature condition for the fluid domain and to specify a heat flux for the solid domain iteratively [20]. Within the physical time step $\mathrm{N}$ and marching from $\mathrm{CHT}$ coupling cycle $\mathrm{i}$ to $\mathrm{i}+1$, the fluid domain is solved first with 
the Dirichlet condition provided from the solid solution, followed by a solid domain solution with the Neumann condition provided from the fluid one:

$$
\begin{gathered}
\left(T_{f}\right)_{i+1}^{N}=\left(T_{S}\right)_{i}^{N} \\
\left(q_{S}\right)_{i+1}^{N}=\left(q_{f}\right)_{i+1}^{N}
\end{gathered}
$$

When the CHT coupling cycles converge, Eqs. 8 and 9 will be a direct manifestation of the physical temperature and flux continuity across the interface.

\section{UNSTEADINESS MODELLING FOR NATURAL CONVECTION (FLUID-DOMAIN ONLY)}

\section{3-1 Computational Set-up and Grid Sensitivity}

Three cases with concentric cylindrical configurations (the first two in 2D and the third in 3D) have been analysed. The first test case is for a simple unsteady natural convection in a 2D annulus domain. The configuration of the problem is shown in Fig.1. The annulus is filled with air. The inner and outer walls of the annulus are isothermal. Initially, the fluid is in a thermally steady-state from a previous steady-state natural convection solution, a cooling process is achieved by linearly decreasing the inner wall temperature to a specific temperature over a certain time and the wall temperature is then maintained at that value.

The fluid domain has an outer diameter (OD) of $1 \mathrm{~m}$ and an inner diameter (ID) of $0.5 \mathrm{~m}$. The inner and outer walls are subject to initial (at $\mathrm{t}=0$ ) isothermal temperature conditions of $\mathrm{T}_{\mathrm{i}}=800 \mathrm{~K}, \mathrm{~T}_{\mathrm{o}}=400 \mathrm{~K}$. The RANS solver is used for the fluid domain with the k- $\varepsilon$ turbulence model. The fluid properties for air are calculated at $600 \mathrm{~K}$. The values of dynamics viscosity, thermal conductivity and specific heat are 3.017 $\mathrm{x} 10^{-5} \mathrm{~kg} / \mathrm{m} \mathrm{s}, 0.04661 \mathrm{~W} / \mathrm{m} \mathrm{K}, 1.0511 \mathrm{~kJ} / \mathrm{kg} \mathrm{K}$, respectively. The Prandtl number is 0.68 . For the natural convection in an annulus, the Rayleigh number based on the gap-width is equal to $2.64 \times 10^{7}$. The pressurebased solver is selected. Typically, 20 sub-iterations are taken for each physical time step in a direct solution. 


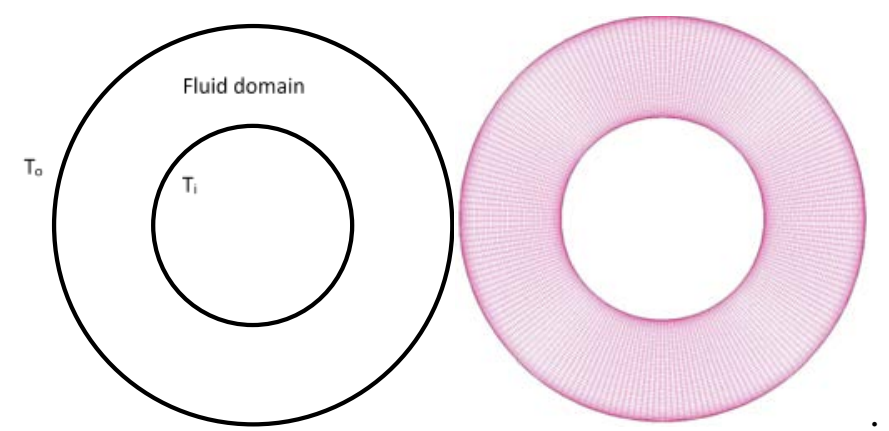

Fig. 1, Computational Domain and Mesh (fluid-domain only).

Grid-dependency tests are carried out first to ensure that a sensible mesh density is chosen so that the results are mesh independent. The details of the different meshes tested are given in Table 1, where the average wall heat fluxes on the inner and outer walls are presented. It can be seen that good grid independent results can be obtained when the number of the total mesh cells is equal to or above 22500 $(300 \times 75)$. This mesh density has been chosen for subsequent analyses.

Table 1. Heat Flux with Different Mesh Sizes

\begin{tabular}{|l|l|l|l|l|}
\hline Grid Size & $100 \times 25$ & $200 \times 50$ & $300 \times 75$ & $400 \times 100$ \\
\hline Heat Flux (ID) & 1382.6 & 1372.8 & 1395.9 & 1395.1 \\
\hline Heat Flux (OD) & -691.3 & -686.4 & -698.0 & -697.5 \\
\hline
\end{tabular}

\section{3-2 Time-Step Restriction of Baseline Flow Solver}

As discussed in the introduction, common flow solvers in commercial codes have been previously shown to exhibit severe restrictions on time step size due to the numerical stability requirement of the solvers for unsteady natural convection. We would thus like to see how restrictive this might be for a URANS based natural convection prediction for configuration dimensions and conditions relevant to steam turbine flexible operations. The computations are first performed with the baseline flow solver, i.e. 'as it is' in FLUENT, using different physical time steps. After the steady natural convection is obtained for the initial condition, the transient process is initiated by changing the inner wall temperature linearly from $800 \mathrm{~K}$ to $600 \mathrm{~K}$ in 10 seconds, which is then kept at $600 \mathrm{~K}$.

The sensitivity of the solution convergence to the time step size $\Delta t$ is illustrated in Figure 2. The solutions are all started from the same initial flow. We look at how the residuals vary with sub-iterations when different physical time steps are adopted. For a small time, step $(\leq 0.2 \mathrm{~s})$ the numerical procedure 
marches in a stable fashion. At each physical time step, the residuals are shown to drop with the subiterations, and the solutions are driven to converge. However, for larger time steps ( $\geq 0.2 \mathrm{sec}$ ), the numerical solutions exhibit a clear divergence behaviour.

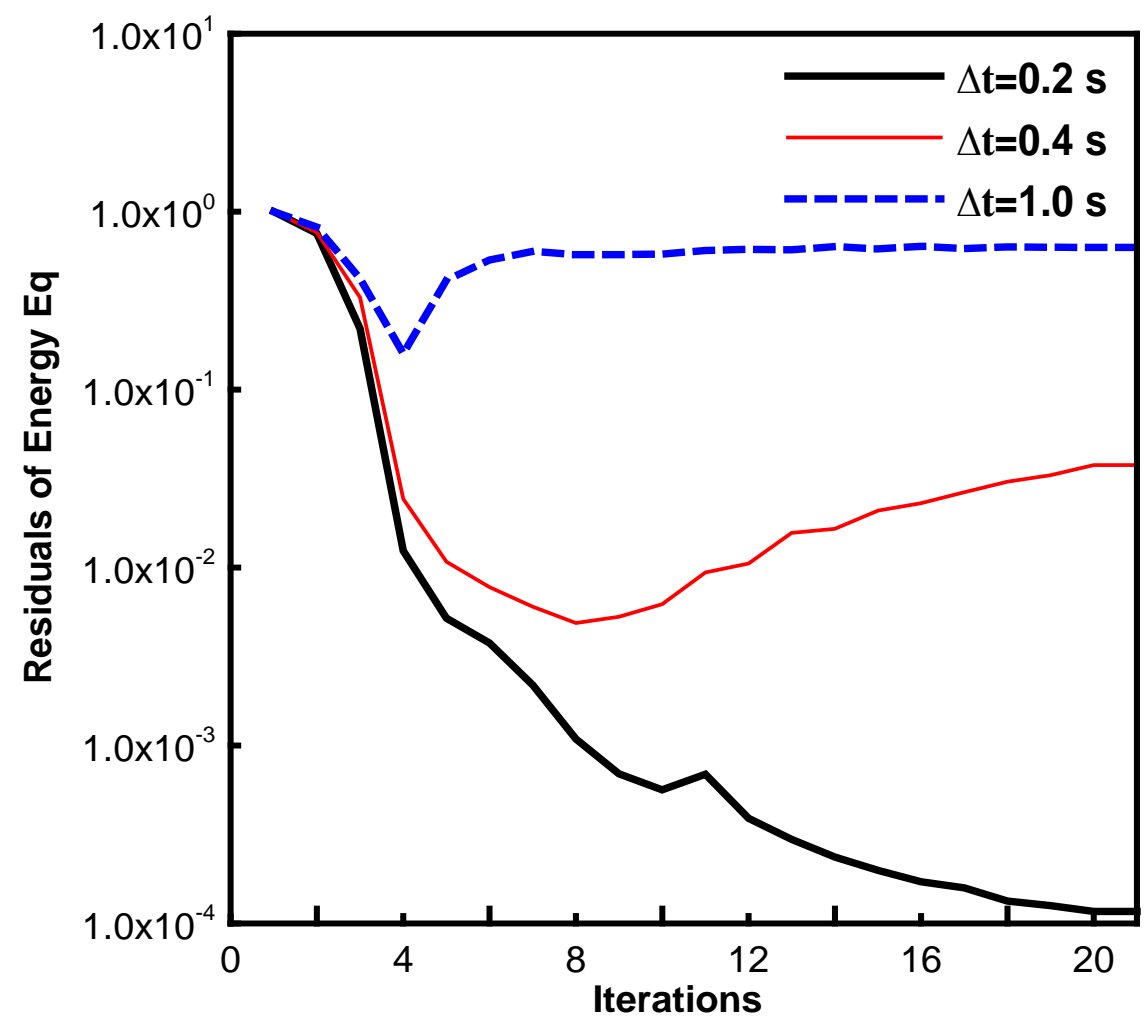

Fig. 2, Residual histories for different time step sizes (Direct unsteady solver of FLUENT).

\section{3-3 Present Source Term Based Dual Time-Stepping Solutions with Large Time Steps}

The severe time step restrictions of the baseline flow solutions can now be contrasted with the much larger time step sizes usable when the present source term based dual time stepping procedure is adopted. For this case, the present results have shown no upper limit of the time step size. Figure 3 indicates the convergence characteristics of the present solver. For the range of time step sizes between $1 \mathrm{~s}$ and $100 \mathrm{~s}$, no convergence issues are indicated.

Having identified the completely different usable time step range for the present flow solver, we now need to first establish if the present method does produce valid unsteady solutions. We address this basic validation requirement by comparing the present solutions with the baseline direct solutions, using different time step sizes for the transient natural convection when the inner surface temperature is changed linearly in time from $800 \mathrm{~K}$ to $600 \mathrm{~K}$. In this case, the linear change of the inner wall temperature is 
specified to occur in different lengths of time corresponding to different sizes of the time steps used in the present solver. Here we only present the results for three time steps sizes, $\Delta \mathrm{t}=10 \mathrm{~s}, 40 \mathrm{~s}$ and $160 \mathrm{~s}$. Thus, the corresponding inner boundary temperature varies linearly in time at a rate of $100 \mathrm{~K} / 10 \mathrm{~s}, 100 \mathrm{~K} / 40 \mathrm{~s}$, and 100K/160s respectively. The wall heat flux distributions at the end of the time periods are shown in Fig.4, and compared to the corresponding direct solutions. For the direct solutions, the time step limits are shown to be independent of the temporal gradient of the wall thermal conditions. So, all the three direct solutions are obtained at a physical time step of $0.2 \mathrm{~s}$ which is the maximum time step size allowed by the numerical stability. We can see the excellent agreement between the present source term solutions and the direct solutions, though with hugely different time step sizes. In Fig.5 and Fig.6, the instantaneous temperature and velocity contours are compared for $\mathrm{t}=10 \mathrm{~s}$ and $160 \mathrm{~s}$ respectively. Clearly both field solutions are also in excellent agreement.

In terms of computing effort, the direct solution for a 10 s period needs 1000 iterations (a physical time step of $0.2 \mathrm{~s}$ with 20 sub-iterations per step). The present solution for the same physical time (one step) needs about 100 iterations. So, for this case, the present solution is 10 times faster than the direct solution. This is already quite considerable. When the time step of $160 \mathrm{~s}$ is taken, the number of sub-iterations required is increased to 400-500. But this increase is not proportional to the increase in the physical time step size, thus the net gain in computational effort then becomes a factor of 32-40.

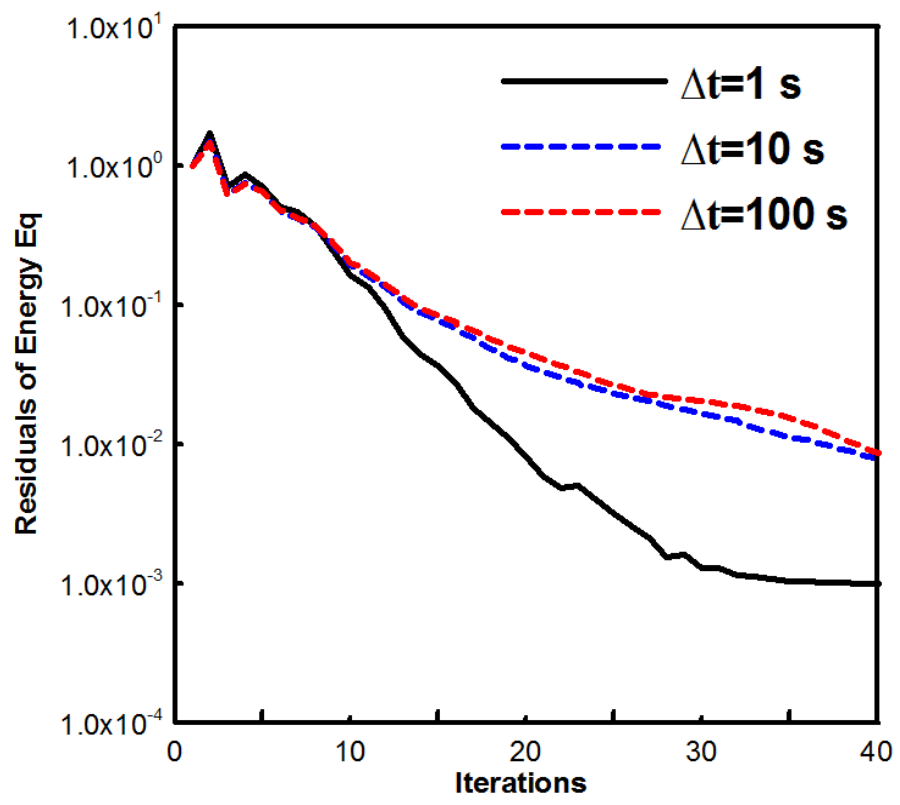

Fig. 3, Residual histories for different time step sizes (Present source term based unsteady solver). 


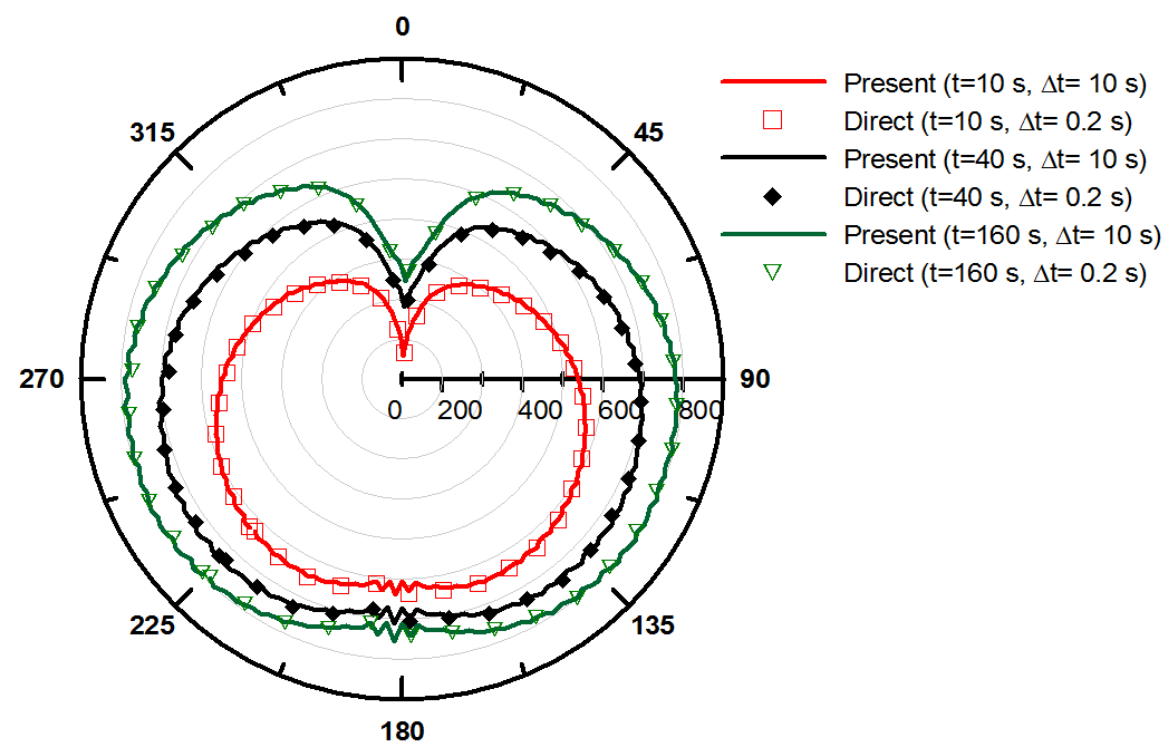

Fig. 4, Comparisons of wall heat fluxes (in $\mathrm{W} / \mathrm{m}^{2}$ ) along circumference (in ${ }^{\circ}$ ) between the present method with large time steps $(\Delta \mathrm{t}=10 \mathrm{~s}, 40 \mathrm{~s}, 160 \mathrm{~s})$ and the direct method of FLUENT with a stability restricted small time step $(\Delta \mathrm{t}=0.2 \mathrm{~s})$.

Clearly, we shall need to consider the temporal errors for the cooling process particularly for the situation where the temporal gradient is higher as at the start of a transient process. Overall, the present results clearly illustrate that time step sizes can be taken much bigger than those of the baseline FLUENT solutions whist still giving an excellent accuracy. And because of this much-enlarged time step range; the corresponding trade-off between temporal accuracy and computational efficiency can now be considered. 


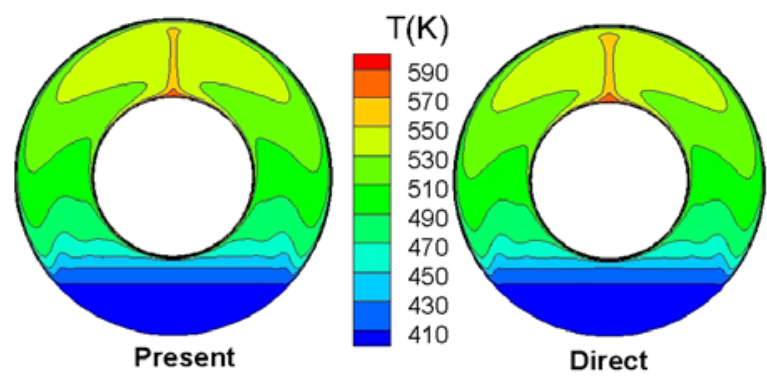

(a) $\mathbf{t}=\mathbf{1 0} \mathrm{s}$.

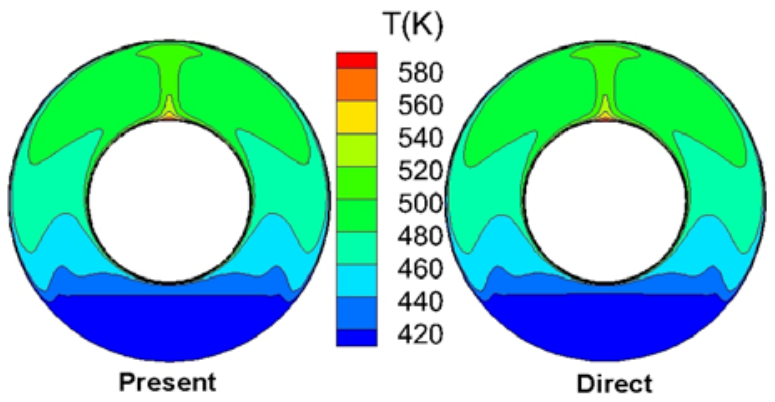

(b) $\mathbf{t}=\mathbf{1 6 0} \mathrm{s}$.

Fig. 5, Instantaneous temperature fields for the Present solutions ( $\Delta \mathrm{t}=10 \mathrm{~s}, 160 \mathrm{~s}$, respectively) and the Direct baseline solutions ( $\Delta \mathrm{t}=0.2 \mathrm{~s}$ for both cases).
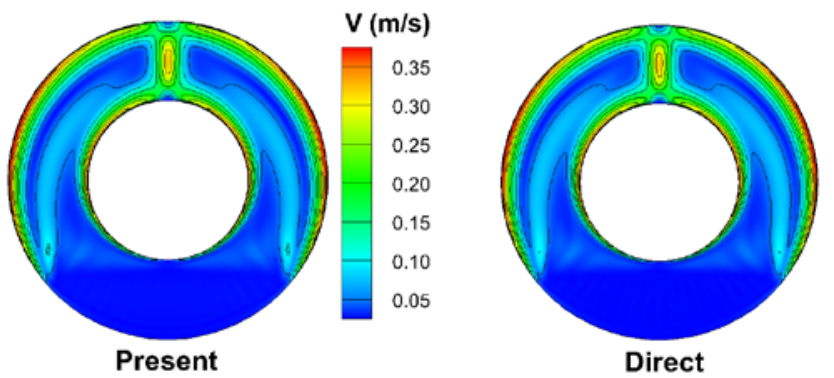

(a) $\mathbf{t}=\mathbf{1 0} \mathrm{s}$.
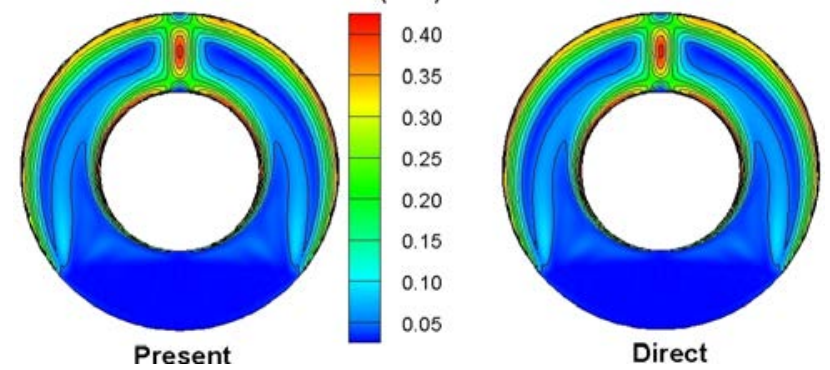

(b) $\mathbf{t}=\mathbf{1 6 0} \mathrm{s}$.

Fig. 6, Instantaneous velocity fields for the Present solutions ( $\Delta \mathrm{t}=10 \mathrm{~s}, 160 \mathrm{~s}$ respectively) and the Direct baseline solutions ( $\Delta \mathrm{t}=0.2 \mathrm{~s}$ for both cases). 


\section{3-4 Impact of Quasi-Steady Flow Model}

Having identified the levels of differences in terms of the time step size, we now examine the impact of using a quasi-steady model for natural convection. A quasi-steady model can be easily realized in the present procedure by simply switching off the temporal gradient term (i.e. the UDF source term in Eq. 6), when the fluid solution proceeds in the physical time, subject to corresponding transient boundary conditions in time. Here, we consider the solution behaviour when the boundary condition is subject to two different temporal gradients. In the first case, the boundary temperature is linearly varied by $20 \mathrm{~K}$ in $0.2 \mathrm{~s}$. In the other, the gradient is reduced by a factor of 4 . It is $5 \mathrm{~K}$ in $0.2 \mathrm{~s}$.

Figure 7 shows the instantaneous heat flux distributions with the different models for the high temporal gradient case. The impact of the temporal errors associated with the quasi-steady model are clearly shown to be non-negligible for this case. Note that for the low gradient case (Fig.8), although the instantaneous heat flux distributions tend to show only small differences, the accumulated effects at a later time may be much larger. The transient load in the solid domain at a later time will depend on the total integrated heat transferred across all the boundaries during the entire time period prior to that time instant. Thus, care will have to be taken with a quasi-steady fluid model for predicting transient thermal loads, even for some rapid ramping in a very short period of time in an otherwise seemingly slow process.

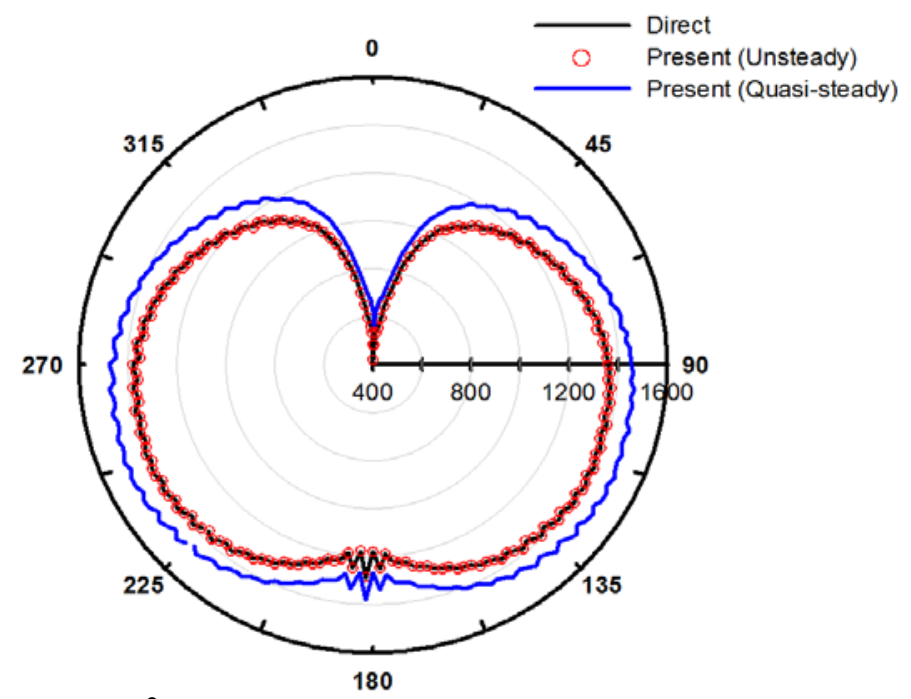

Fig. 7, Wall heat fluxes $\left[\mathrm{W} / \mathrm{m}^{2}\right]$ along circumference (boundary temperature gradient of 20K/0.2s)

It should also be pointed out that the present method including the unsteady source terms should consume largely the same amount of computing time as a quasi-steady transient approach without the unsteady fluid source term. Thus, the present method does have a clear advantage with a much-expanded 
validity at little or no extra cost compared to a quasi-steady model, particularly when integrated to a loosely coupled CHT procedure.

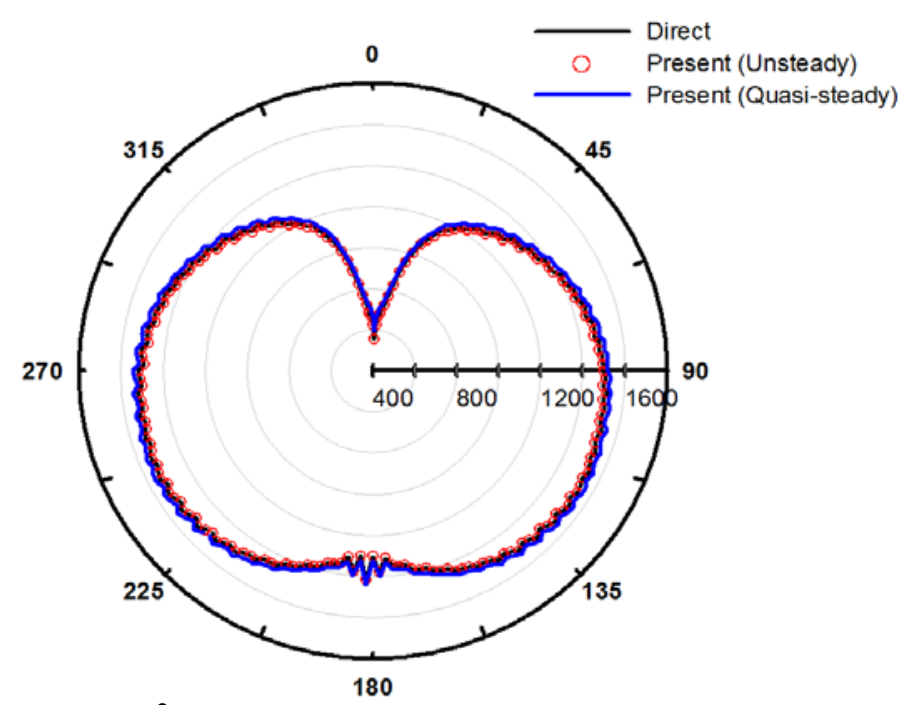

Fig. 8, Wall heat fluxes $\left[\mathrm{W} / \mathrm{m}^{2}\right]$ along circumference (boundary temperature gradient of 5K/0.2s)

\section{UNSTEADINESS MODELLING CHARACTERISTICS FOR NATURAL CONVECTION (FLUID-SOLID CHT)}

\section{4-1 Steady CHT for Coupled Annular Fluid-Solid Domains}

The first conjugate heat transfer test case is for a simple 2D concentric annulus domain configuration as shown in Figure 9. The fluid domain is the same as that used for the fluid-only analyses. The outer boundary of the fluid domain is an isothermal wall at $400 \mathrm{~K}$. The solid domain has an inner diameter (ID) of $350 \mathrm{~mm}$, and its outer boundary is the interface with the fluid domain. The inner boundary of the solid domain is an isothermal wall at $800 \mathrm{~K}$. The solid material is taken to be steel with a thermal conductivity of $16.3 \mathrm{~W} / \mathrm{m} \cdot \mathrm{K}^{-1}$.

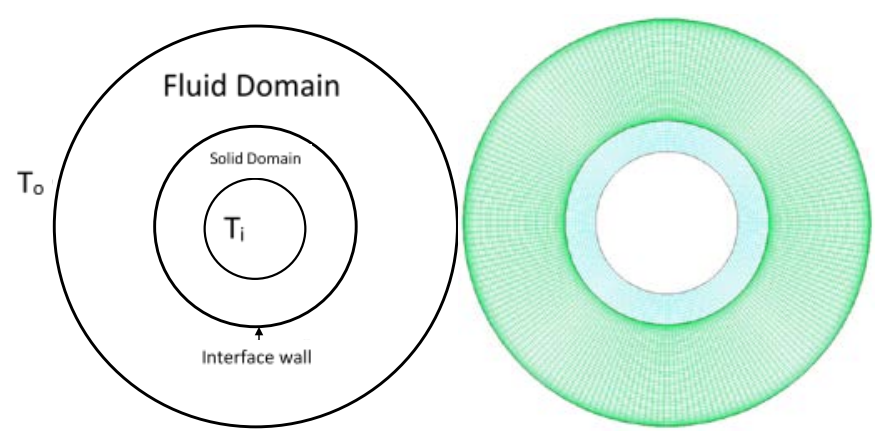

Fig. 9, Fluid and solid domains and mesh. 
The present loosely coupled CHT solution is compared to the direct fully coupled CHT solution of FLUENT, firstly for a steady natural convection. Figure 10 shows temperature distributions at the interface by the present loosely coupled method, compared to those calculated by the direct fully coupled CHT of FLUENT. It can be seen that the loosely coupled and the direct fully coupled CHT solutions are in excellent agreement. Figure 11 shows the wall heat fluxes on both the solid and fluid sides of the interface, they are practically identical. Given the sequential updating of temperature and heat flux for the two sides of an interface for the present loosely coupled approach (see Eqs.8, 9), the heat fluxes on both sides of the interface should match each other exactly, which is a clear and consistent manifestation of the physical interface condition.

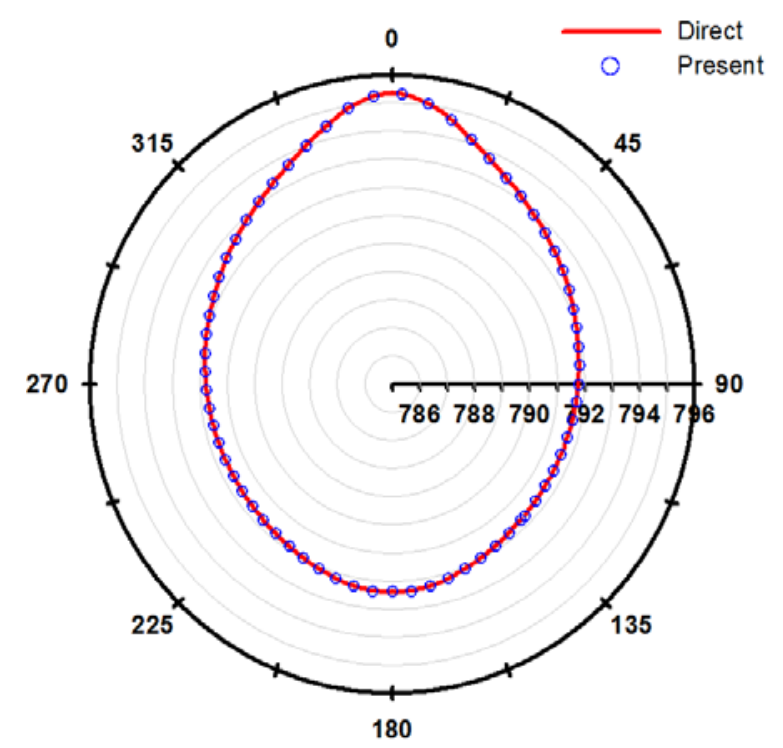

Fig. 10, Comparison of fluid-solid interface temperatures [K] between the present loosely coupled CHT ('Present') and the direct fully coupled CHT ('Direct') solutions. 


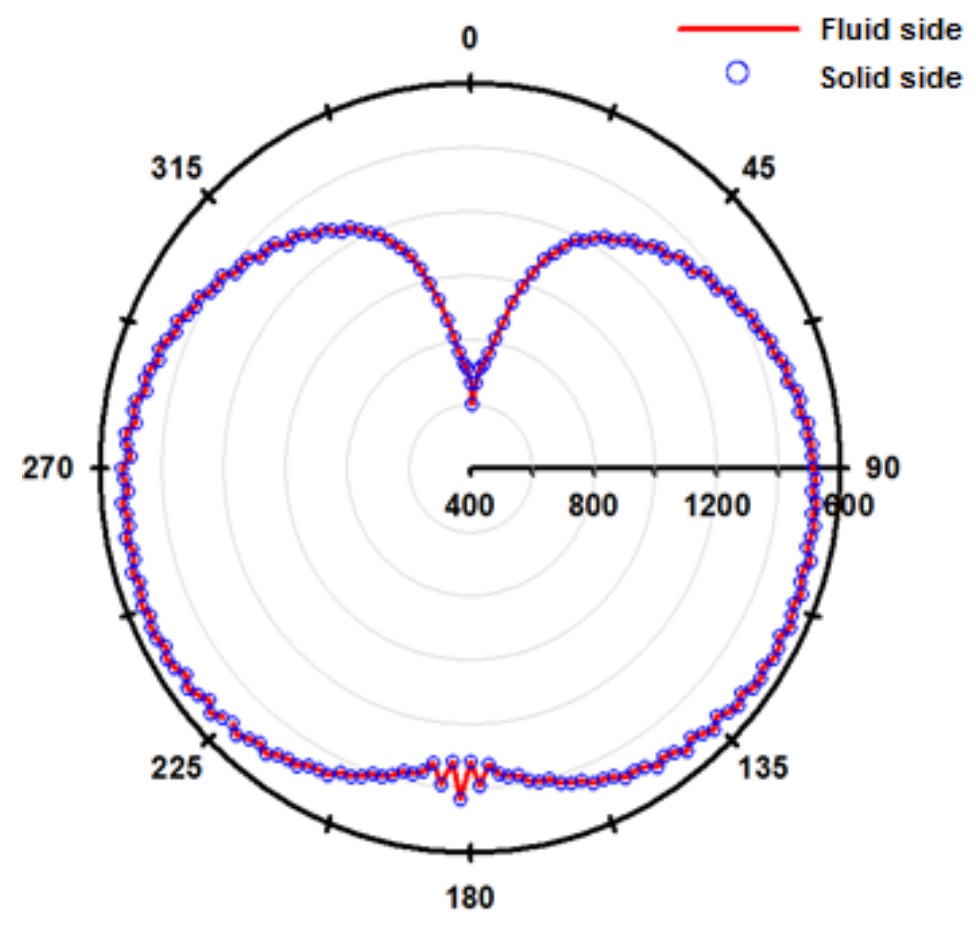

Fig. 11, Heat fluxes $\left[\mathrm{W} / \mathrm{m}^{2}\right]$ on both sides of interface

\section{4-2 Usable Time Step Sizes for Transient CHT Solution}

For the fluid domain natural convection analyses, we have seen clearly a maximum time step restriction due to the stability of the baseline flow solver. For a transient CHT analysis, we would like also to check first if the time step restriction is changed by coupling with a solid domain.

For a transient natural convection process, the inner wall of the solid domain is now subject to a linear decrease in temperature ( $800 \mathrm{~K}$ to $600 \mathrm{~K})$ in $10 \mathrm{~s}$, and then kept at $600 \mathrm{~K}$.

Numerical tests are conducted for the direct CHT solver in FLUENT by using $\Delta t=0.2 \mathrm{~s}, 0.4 \mathrm{~s}, 0.5 \mathrm{~s}, 1.0 \mathrm{~s}$ and $10 \mathrm{~s}$. It is observed that the solutions again can only be stable for a time step less than $0.2 \mathrm{~s}$. Figure 12 shows the convergence histories of the direct solver with different time steps sizes. In clear contrast, Figure13 shows the convergence histories of the present loosely coupled procedure. The results confirm that the time step limit is more or less the same as that for the fluid-domain only case.

Thus, the results of the present cases indicate that it is the numerical instability of the flow solver that completely dictates the allowable time step size, independent of the fluid-solid CHT coupling. The test results also suggest that the time step restriction on the baseline flow solver should remain largely the same regardless of the temporal gradient of the flow field. 


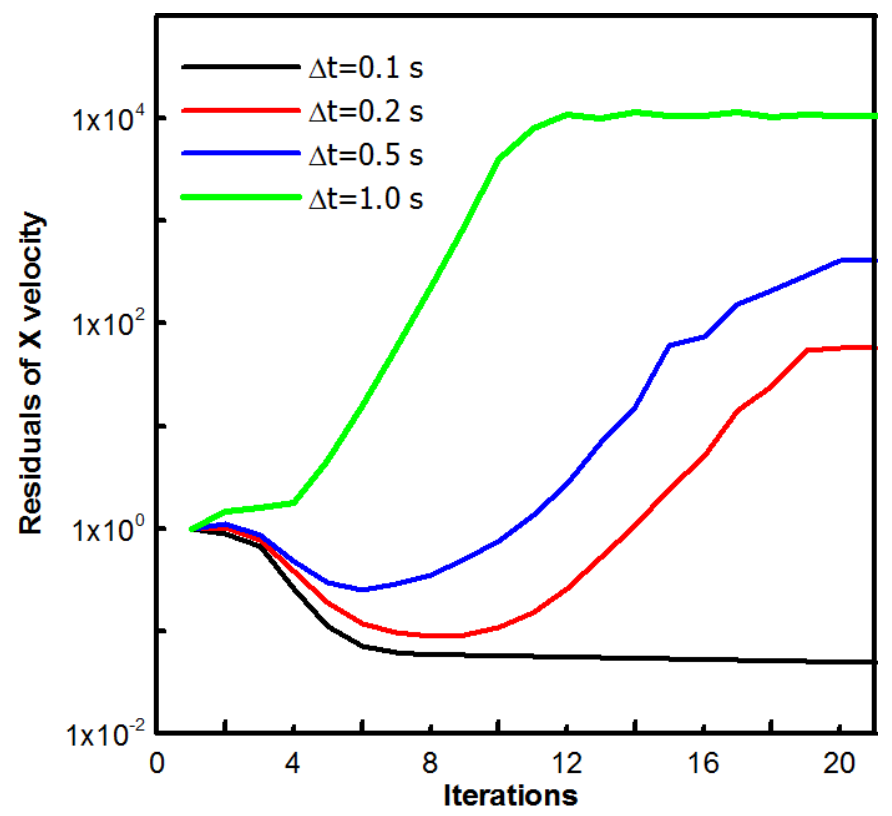

Fig. 12, Convergence histories of the baseline direct coupled CHT solutions for different time steps.

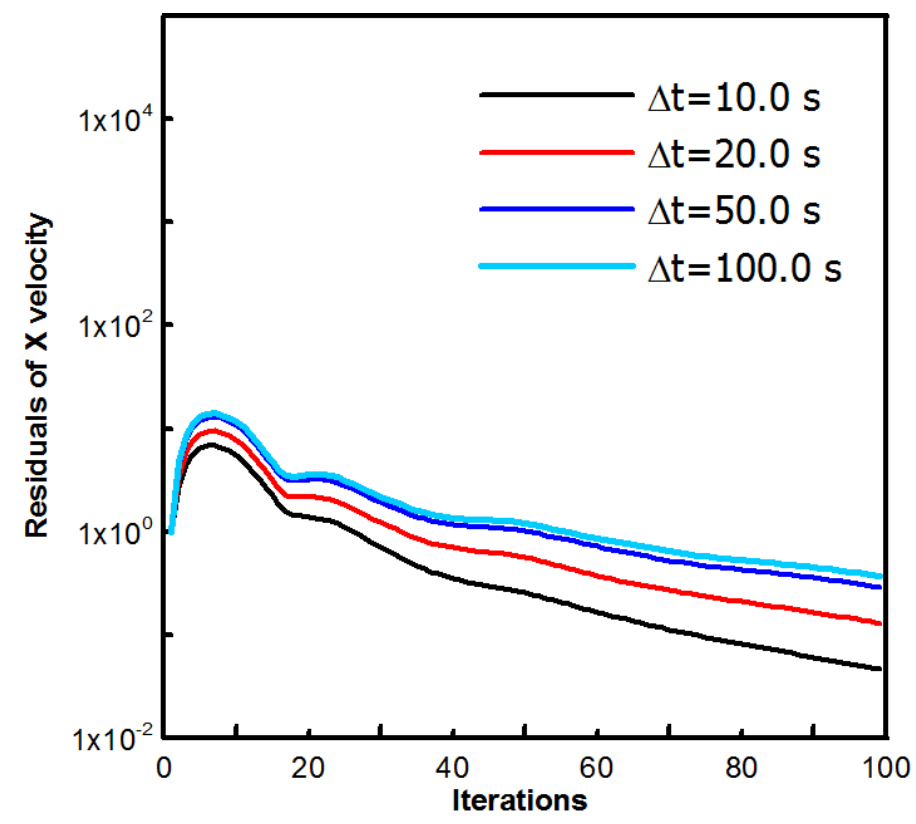

Fig. 13, Convergence histories of the present loosely coupled CHT solutions for different time steps.

On the other hand, the present loosely coupled CHT methods shows no upper limit on the time steps sizes, similarly to the fluid-domain only cases, e.g. as shown Fig.3. The stark contrast can have some considerable implications on the computational efficiency comparison as will be discussed in the following section. 


\section{4-3 Effect of Time Step Size on Accuracy and Speed of Loosely Coupled CHT Solution}

Now we examine the conjugate heat transfer capability of the present loosely coupled CHT implementation for transient natural convection. We first look at the effect of time step size on both accuracy and stability of simulation results for the given transient wall temperature variation (thus the same temporal gradient). The loosely coupled CHT solution is obtained for matching in time at different physical time steps to check the accuracy and stability of the simulation results. For consistency of the comparisons, all solutions are restarted from the same initial solution with $\mathrm{Ti}=600 \mathrm{~K}$ obtained at the end of the $10 \mathrm{~s}$ period of the linear variation of the inner solid wall boundary temperature.

Figure 14 shows the effects of different time step sizes on the numerical predictions of wall temperature distributions at $t=700 \mathrm{~s}$. Here, the physical time step sizes of $10 \mathrm{~s}, 12.5 \mathrm{~s}, 15 \mathrm{~s}$, and $20 \mathrm{~s}$ are examined respectively. These solutions are compared with the direct CHT solution at the same time instant but marched at a much smaller time step due to the stability requirement ( $\Delta \mathrm{t}=0.2 \mathrm{~s}$ ). It can be seen that for this initial stage of the transient natural convection process of a relatively large temporal gradient, the loosely coupled solutions agree well with the direct CHT solution if a physical time step of $10 \mathrm{~s}$ is used. The solution accuracy deteriorates with larger time steps, as expected.

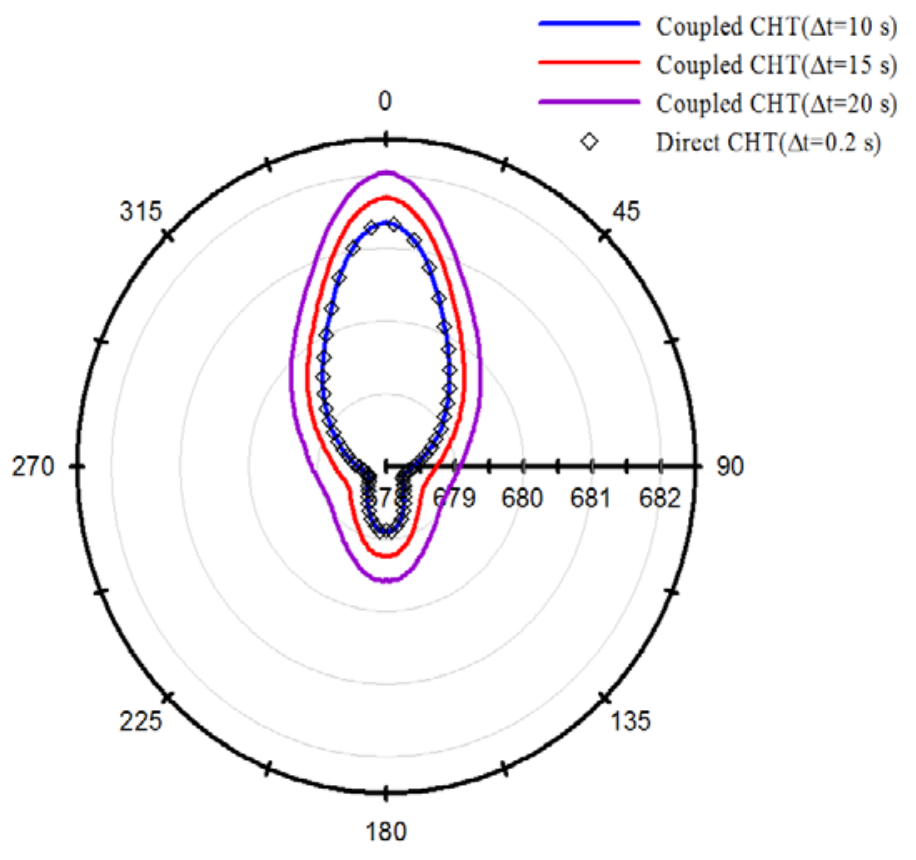

Fig. 14, Interface temperatures [in $\mathrm{K}$ ] at $\mathrm{t}=700 \mathrm{~s}$ by the Direct CHT method with a small-time step $(\Delta t=0.2 \mathrm{~s})$ and the present loosely coupled CHT method with different time steps. 
A long-term evolution and the validity of the present CHT coupling can be illustrated by instantaneous interface temperature distributions at different instants in time. In Figure 15, temperature variations along the solid-fluid interface are compared between the direct solution $(\Delta t=0.2 \mathrm{~s})$ and the present loosely coupled solution ( $\Delta \mathrm{t}=10 \mathrm{~s})$, plotted for different time instants. The comparison between the direct coupled $\mathrm{CHT}$ and the loosely CHT shows very good agreement for all the time instants. Temperature contours for fluid and solid domains corresponding to $t=500 \mathrm{~s}$ and $1000 \mathrm{~s}$ respectively are shown in Figure 16. In both cases the results by the present loosely coupled CHT and the direct CHT methods are in excellent agreement.

In terms of the computational efficiency, the loosely coupled CHT typically needs only 1-2 coupling cycle at each time step. Given the difference in the time step sizes (10 s vs $0.2 \mathrm{~s}$ ), and the number of subiterations needed, the present loosely coupled CHT is about 10 times faster than the direct CHT method.

A further point worthwhile making is regarding the prospect of using an adaptive time stepping in predicting a transient natural convection, particularly in relation to the typically long-time scales relevant to a steam turbine natural cooling process. It is noted that for the present loosely coupled CHT procedure, there is practically no upper time step limit. Also, we make an observation that the most stringent temporal accuracy requirement would typically occur at the initial stage of the process. Hence in terms of the computational accuracy, our maximum time step will be confined by that at the initial stage, whilst we can make a far bigger computational efficiency gain if we use larger time steps at a later stage of the cooling process. 


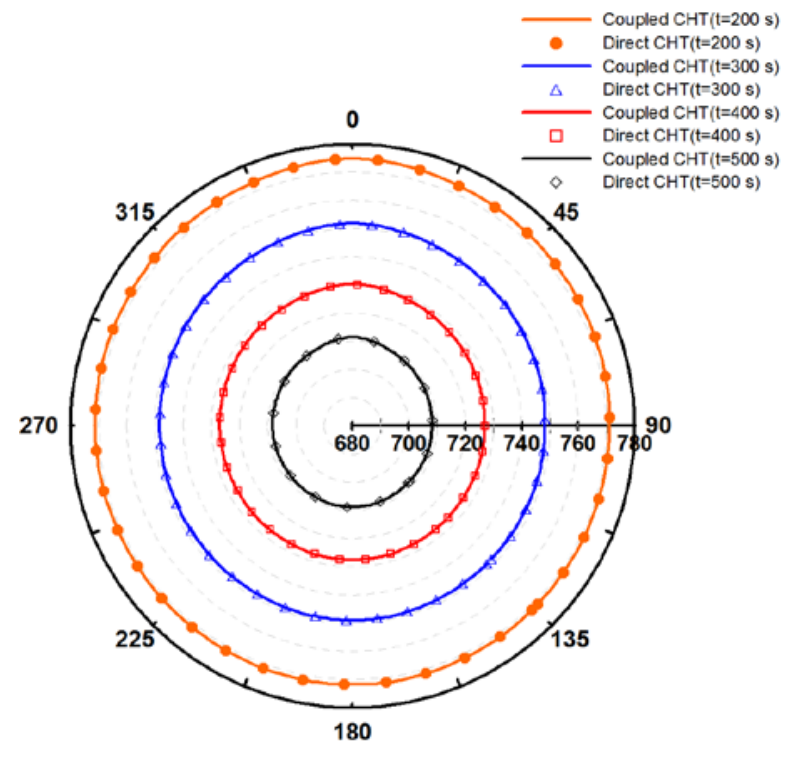

(a) 200s-500s

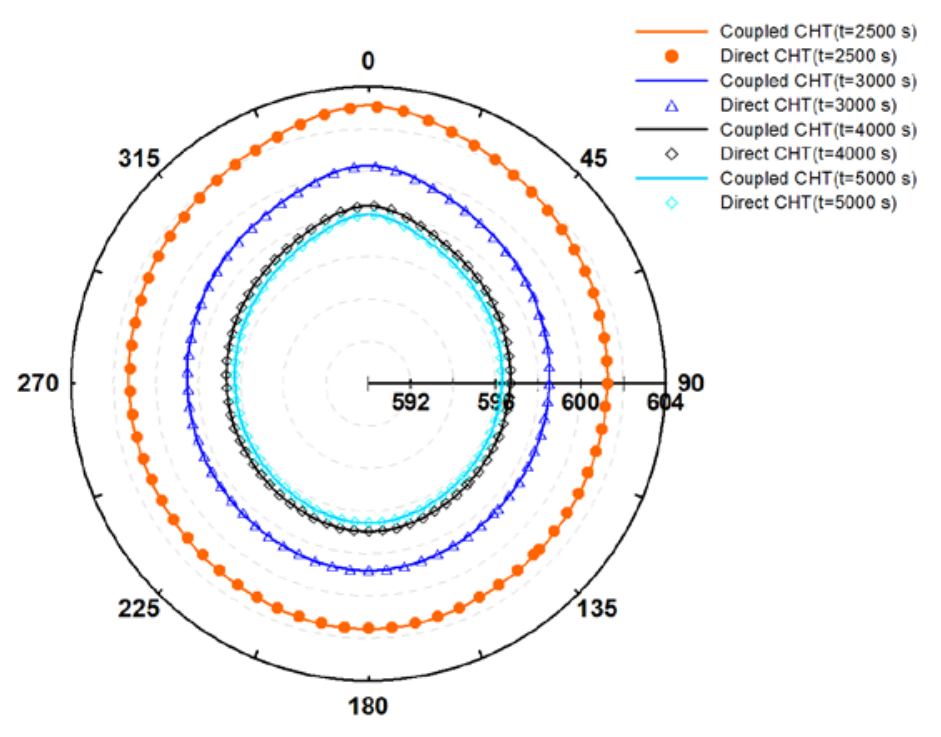

(b) $2500 \mathrm{~s}-5000 \mathrm{~s}$

Fig. 15, Time evolution of interface temperature profiles [K] (Direct CHT: $\Delta \mathrm{t}=0.2 \mathrm{~s}$; Loosely Coupled CHT: $\Delta \mathrm{t}=10 \mathrm{~s}$ ). 


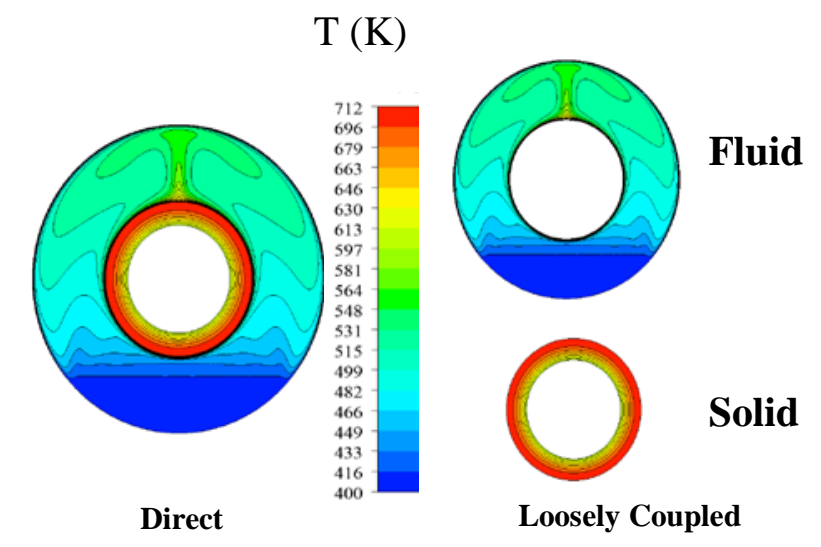

(a) $\mathbf{t}=500 \mathrm{~s}$

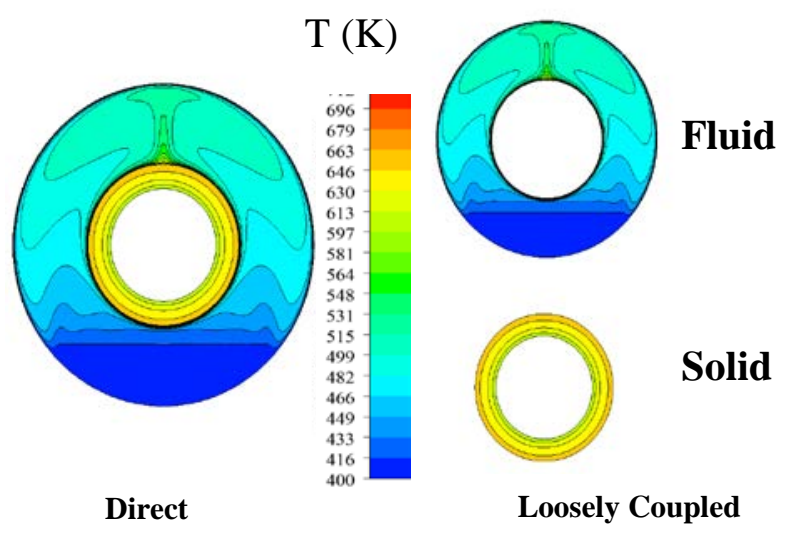

(b) $\mathbf{t}=\mathbf{5 0 0 0}$

Fig. 16, Comparison of temperature contours in solid and fluid domains between the direct solution $(\Delta \mathrm{t}=0.2 \mathrm{~s})$ and the present solution $(\Delta \mathrm{t}=10 \mathrm{~s})$ at $\mathrm{t}=500 \mathrm{~s}$ and $\mathrm{t}=1000 \mathrm{~s}$, respectively.

To illustrate the point regarding an adaptive time stepping, we take the pre-run solution at $\mathrm{t}=2000 \mathrm{~s}$ as the initial field and then compare the solutions marched to $\mathrm{t}=3000 \mathrm{~s}$ with different time steps (Fig. 17). We also check the solutions with different time step sizes marched from 3000 s to $4000 \mathrm{~s}$, shown in Fig. 18. It is clear that we can use much larger time steps with good accuracy at these later stages. For the phase from $3000 \mathrm{~s}$ to 4000s, a time step size of $100 \mathrm{~s}$ gives the results as accurate as those by the direct solution using a time step of $0.2 \mathrm{~s}$. Thus, for this period, the time step can be enlarged by 500 times relative to the direct solution.

It is reminded that the time step limit for the direct CHT is shown to hardly change with the temporal gradient, i.e. a time step of $0.1 \sim 0.2 \mathrm{~s}$ will still have to be used here for the direct CHT method. Thus, a difference in time step size for the later stage of a cooling process can be of a factor of $\sim 10^{3}$. Based on these results and discussions, we can thus see a very strong case for adopting a loosely coupled CHT 
procedure for transient natural convection predictions for steam turbine flexible operations and similar applications.

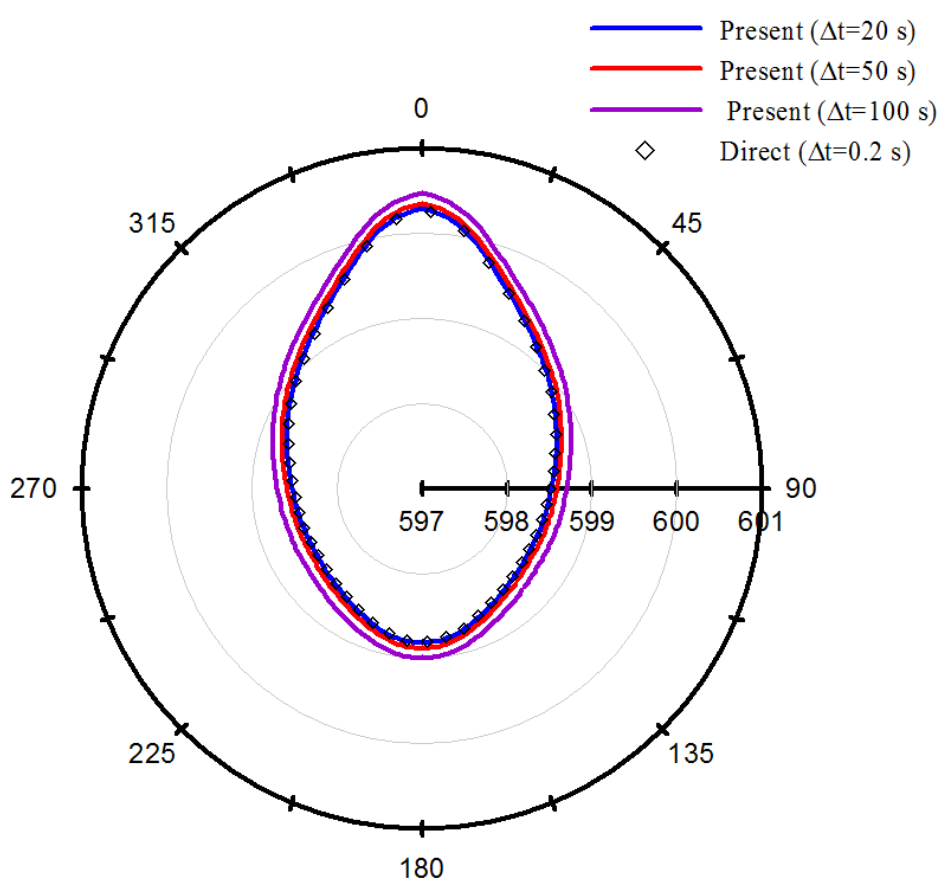

Fig. 17, Effect of the time step sizes on the predicted interface wall temperature [K] distributions $(\mathrm{t}=3000 \mathrm{~s})$.

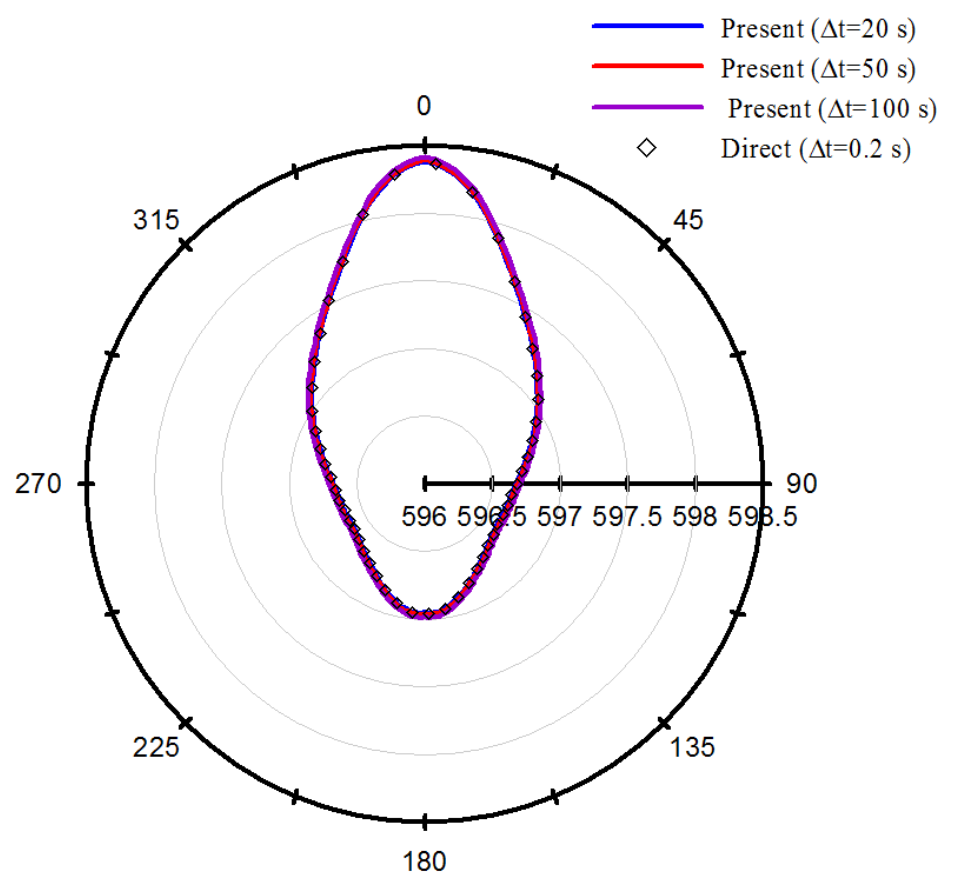

Fig. 18, Effect of the time step sizes on the predicted interface wall temperature $[\mathrm{K}]$ distributions $(\mathrm{t}=4000 \mathrm{~s})$. 


\section{4-4 3-D Transient CHT Case}

So far, the computational case studies are all for 2D configurations. The method also has been implemented and verified for 3D configurations. The final case is a simple extended 3D geometry for the fluid-solid coupled domains from the 2D CHT cases. The configuration is shown in Fig. 19. The axial dimension of the domain is $0.3 \mathrm{~m}$. All boundary and interface conditions on those circumferential surfaces are the same as those in the previous sections. The two outer surfaces of the two side walls in the third dimension are adiabatic. The corresponding inner surfaces of these side walls are subject to the fluid-solid interface treatment.

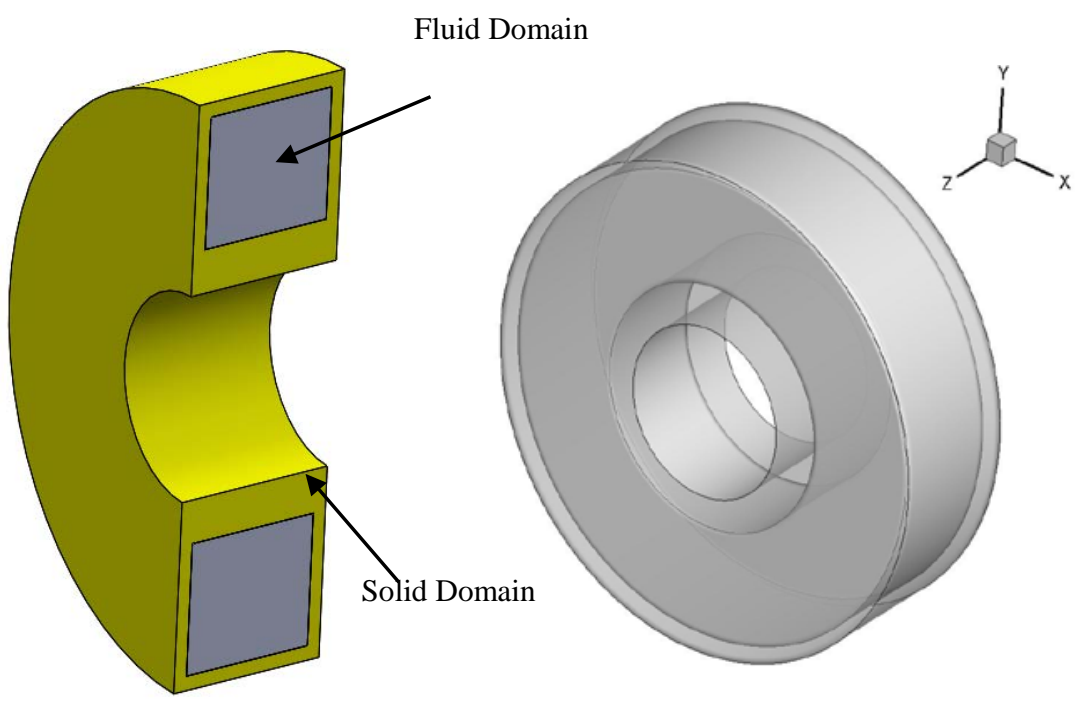

Fig. 19, 3D computational configuration

Figure 20 shows the comparison in instantaneous heat flux on the fluid-solid interface at $t=200 \mathrm{~s}$ between the direct CHT solution ( $\Delta \mathrm{t}=0.2 \mathrm{~s})$ and the present loosely coupled CHT ( $\Delta \mathrm{t}=10 \mathrm{~s})$. The corresponding comparison in the temperature contours between the two solutions is given in Fig.21 for a cut plane normal to the axis at the middle point of the domain.

All the comparisons demonstrate excellent agreement between the present loosely coupled solutions and the baseline direct CHT solutions for this 3D configuration. The hugely different time steps usable for the two methods indicate a similar computational efficiency gain in a 3D case to that in a 2D case. 

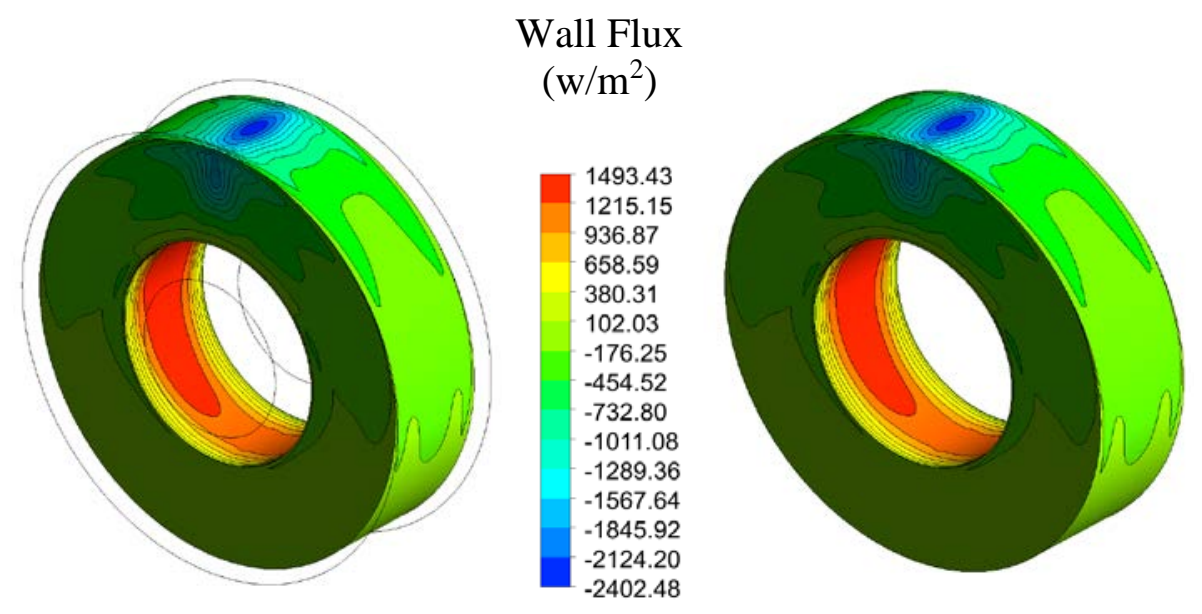

(a) Direct CHT

(b) Loosely Coupled CHT

Fig. 20, Comparison of instantaneous heat flux contours at fluid-solid interface (at $\mathrm{t}=200 \mathrm{~s}$ ), between the direct solution $(\Delta \mathrm{t}=0.2 \mathrm{~s})$ and the present loosely coupled solution $(\Delta \mathrm{t}=10 \mathrm{~s})$.

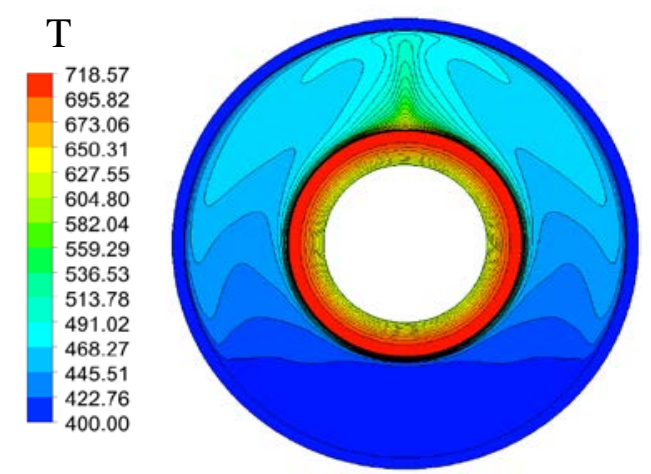

(a) Direct CHT

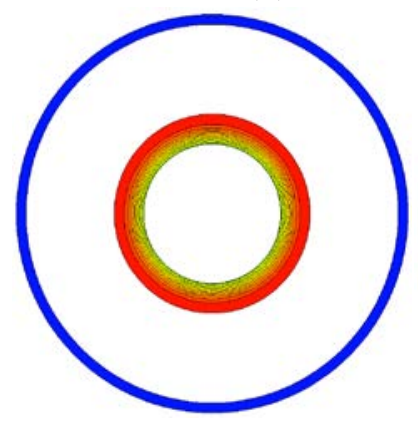

Solid domain

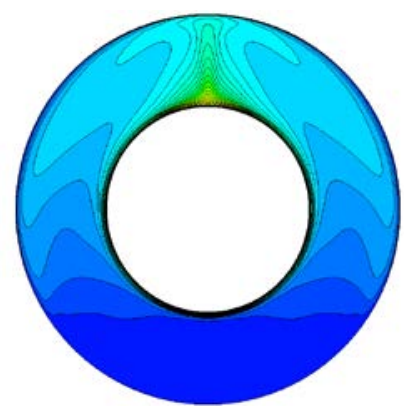

Fluid domain

(b) Loosely Coupled CHT

Fig. 21, Comparison of instantaneous temperatures on a mid-domain cut plane (at $t=200 \mathrm{~s}$ ), between the direct solution $(\Delta t=0.2 \mathrm{~s})$ and the present loosely coupled solution $(\Delta \mathrm{t}=10 \mathrm{~s})$. 


\section{CONCLUSIONS}

Impact of the temporal gradient of unsteady flow and its modelling in relation to numerical stability and modelling accuracy is assessed for the transient natural convection prediction relevant to steam turbine flexible operations. A source term based dual timing formulation is adopted and implemented in a commercial solver. Furthermore, a loosely coupled procedure has been applied, for the first time, to transient conjugate heat transfer predictions for natural convection. The results have consistently demonstrated that the present source-term based unsteady flow solver allows for much larger computational time steps to be used without the stability issues as often encountered in common commercial codes for unsteady natural convection.

The possibility of using a much larger time step size has led to the need to assess the impact of using different time step sizes wholly dictated by the temporal accuracy requirement. Numerical simulations demonstrate that the present method can drastically reduce computational time compared to the baseline method in the commercial code. The present method is also shown to be more accurate at hardly any extra costs compared to a quasi-steady flow model based procedure when unsteady effects are non-negligible. Several 2D and 3D test cases relevant to a natural cooling process of steam turbines are presented for validations and demonstrations.

\section{ACKNOWLEDGMENTS}

The present work is sponsored by UK Engineering and Physical Science Research Council (EPSRC) and GE Power.

\section{REFERENCES}

[1] Topel M, Genrup M, Jöcker M, Spelling J, Laumert B. “Operational Improvements for Startup Time Reduction in Solar Steam Turbines”. ASME J Eng Gas Turb Power, 2015;137(4):042604-042604-8. doi:10.1115/1.4028661.

[2] Born D, Stein P, Marinescu G, Koch S, Schumacher D. “Thermal Modeling of an Intermediate Pressure Steam Turbine by Means of Conjugate Heat Transfer-Simulation and Validation”. ASME J Eng Gas Turb Power,. 2016;139(3):031903-031903-10. doi:10.1115/1.4034513.

[3] Marinescu G, Mohr WF, Ehrsam A, Ruffino P, Sell M. "Experimental Investigation in to Thermal Behavior of Steam Turbine Components-Temperature Measurements with Optical Probes and Natural 
Cooling Analysis”. ASME J. Eng. Gas Turb. Power, 2013;136(2):021602-021602-10. doi:10.1115/1.4025556.

[4] Marinescu G, Stein P, and Sell M. "Natural Cooling and Startup of Steam Turbines: Validity of the Over-Conductivity Function”. ASME J Eng Gas Turb Power, 2015;137(11):112601-112601-9. doi:10.1115/1.4030411.

[5] Maffulli, R., and He, L., 2014, "Wall Temperature Effects on Heat Transfer Coefficient for High Pressure Turbines”, AIAA Journal of Propulsion and Power, 2014; 30:4, 1080-1090

[6] Maffulli, R., and He, L., "Impact of Wall Temperature on Heat Transfer Coefficient and Aerodynamics for 3-D Turbine Blade Passage”. ASME J Thermal Sci. Eng. Appl., 9(4), 041002 (Apr 19, 2017), doi:10.1115/1.4036012.

[7] Zhang Q, and He L. "Impact of Wall Temperature on Turbine Blade Tip Aerothermal Performance". ASME J Eng. Gas Turb. Power, 2014;136(5):052602-052602-9. doi:10.1115/1.4026001.

[8] He L, and Oldfield MLG. "Unsteady Conjugate Heat Transfer Modeling”. ASME. J. Turbomach. 2011;133(3):031022-031022-12. doi:10.1115/1.4001245.

[9] Sun Z, Chew JW, Hills NJ, Volkov KN, and Barnes CJ. "Efficient Finite Element Analysis/Computational Fluid Dynamics Thermal Coupling for Engineering Applications”. ASME. J. Turbomach. 2010;132(3):031016-031016-9. doi:10.1115/1.3147105.

[10] Errera M, and Baqué B., “A quasi-dynamic procedure for coupled thermal simulations”, International Journal for Numerical Methods in Fluids, Vol. 72, Issue 11 (2013) pp: 1183-1206.

[11] Wang Z, Corral R, Chaquet JM, and Pastor G., “Analysis and Improvement of a Loosely Coupled Fluid-Solid Heat Transfer Method”. ASME. Turbo Expo: Power for Land, Sea, and Air, Volume 3B: Heat Transfer: V03BT11A006. doi:10.1115/GT2013-94332.m June 2013.

[12] Altaç, Z., and Uğurlubilek, N., "Assessment of turbulence models in natural convection from twoand three-dimensional rectangular enclosures”, International Journal of Thermal Sciences, Volume 107, 2016, pages 237-246, ISSN 1290-0729.

[13] Ma, J., and Xu, F., Jia Ma, Feng Xu, “Transient Flows around a Fin at Different Positions”, Procedia Engineering, Volume 126, 2015, pages 393-398, ISSN 1877-7058. 
[14] Xu, F., and Saha, S. C., " Transition to an unsteady flow induced by a fin on the sidewall of a differentially heated air-filled square cavity and heat transfer”, International Journal of Heat and Mass Transfer, Volume 71, 2014, pages 236-244, ISSN 0017-9310.

[15] He, L., and Fadl, M., " Multi-scale Time Integration for Transient Conjugate Heat Transfer”. Int. J. Numer. Meth. Fluids, 2017, 83: 887-904. doi: 10.1002/fld.4295.

[16] Perelman, T.L, “On Conjugated Problems of Heat Transfer”, International Journal of Heat and Mass Transfer, Volume 3, Issue 4, 1961, pages 293-303, ISSN 0017-9310.

[17] Jameson, A., “Time-Dependent Calculations using Multi-Grid, with Applications to Unsteady Flows past Airfoil and Wings”, 1991, AIAA Paper 91-1596.

[18] Arnone A., Liou, M.S. Povinelli, LA, "Integration of Navier-Stokes equations using dual time stepping and a multigrid method", AIAA Journal, Vol. 33, No. 6 (1995), pp. 985-990.

[19] He, L., "3D Navier-Stokes Analysis of Rotor-Stator Interactions in Axial Flow Turbines", Proc. IMech.E, Part-A, Journal of Power and Energy, Vol.214, pp13-22, Jan. 2000.

[20] Giles, M.B., "Stability Analysis of Numerical Interface Conditions in Fluid-Structure Thermal Analysis”. Int. J. Numer. Meth. Fluids, 1997, 25: 421-436. 